\title{
Knockdown of Polycomb-Group RING Finger 6 Modulates Mouse Male Germ Cell Differentiation in Vitro
}

\author{
Jin Sun ${ }^{a}$ Jian Wang ${ }^{a}$ Lin He ${ }^{a}$ Yi Linc Ji Wu ${ }^{a, b}$ \\ aKey Laboratory for the Genetics of Developmental \& Neuropsychiatric Disorders (Ministry of \\ Education), Bio-X Institutes, Shanghai Jiao Tong University, Shanghai, 'Key Laboratory of Fertility \\ Preservation and Maintenance of Ministry of Education, Ningxia Medical University, Yinchuan, Ningxia, \\ 'The International Peace Maternity and Child Health Hospital, School of Medicine, Shanghai Jiao Tong \\ University, Shanghai, China
}

\section{Key Words}

PCGF6 $•$ Knockdown • Germ cell development

\begin{abstract}
Background: Polycomb-group RING finger 6 (PCGF6), one of six PCGF homologs, is the core component of the PRC1 complex that plays critical roles in epigenetic transcriptional silencing in higher eukaryotes. However, the biological functions of PCGF6 are unknown. Method: qRT-PCR and Western blot were used to detect the expression profile of PCGF6 in testes. Fluorescent immunohistochemistry was used to examine the cellular localization of PCGF6 protein in testes. Cell proliferation was tested by performing CCK-8 and EdU incorporation assay. Cell cycle and haploid cell population analysis was determined by flow cytometry using propidium iodide DNA staining. Co-immunoprecipitation experiment was conducted using PCGF6 antibody to obtain interacting protein of PCGF6. Luciferase reporter assays were performed to examine the promoter activity of PCGF6 in cells overexpressing OVOL1 and PLZF. Results: PCGF6 was expressed predominantly in meiotic and post-meiotic male germ cells, could negatively regulate the proliferation of GC-2 spd cells, an immortalized mouse spermatogenic cell line, and could modulate the differentiation of GC-2 spd cells in vitro. PCGF6 could indirectly interact with HSPA2, a key factor that is essential for male meiosis, and OVOL1 and PLZF, two key transcription factors that are involved in spermatogenesis, could positively and negatively modulate Pcgf6 promoter activity, respectively. PCGF1, BMI1, and PCGF5 are also highly expressed in mouse testes like PCGF6. Conclusion: PCGF6 may play important roles in male germ cell development.
\end{abstract}




\section{Introduction}

Polycomb group (PcG) proteins are epigenetic repressors that are required to preserve transcriptionally silenced states through epigenetic marking of target genes in higher eukaryotes, and they play essential roles in cell differentiation and development [1]. They function within multiprotein complexes via action on chromatin; these complexes are called Polycomb repressive complexes (PRCs). Polycomb repressive complex 1 (PRC1), one of the two major classes of PRCs identified in mammals, is generally responsible for chromatin compaction and monoubiquitination of histone $\mathrm{H} 2 \mathrm{~A}$, and there are several variants that are composed of different homologs of core components and noncanonical PRC1 members [2, 3]. PcG RING finger (PCGF) homologs (PCGF1, PCGF2, PCGF3, BMI1, PCGF5, and PCGF6) are critical core components in the assembly of distinct PRC1-related complexes, and they all have also been found to associate with ring finger protein 2 (RNF2). The RNF2-PCGF heterodimer is catalytically competent as an E3 ubiquitin transferase and is the scaffold for the assembly of additional components [4-6]. An increasing number of studies have demonstrated that the mammalian PCGFs play important roles in cell proliferation, differentiation, and tumorigenesis. For instance, PCGF1, also known as NSPc1, could promote tumor cell cycle progression and cell proliferation and also is necessary for the terminal differentiation of hematopoietic cells [7, 8]. In contrast, PCGF2, also known as Mel-18, could inhibit the tumor cell proliferation and self-renewal activity of hematopoietic stem cells $[9,10]$. Additionally, it is noteworthy that BMI1, also known as PCGF4, is a famous oncogene and stem cell selfrenewal factor that is extensively involved in the self-renewal of embryonic stem cells and adult stem cells, including neural stem cells, hematopoietic stem cells, spermatogonial stem cells, lung epithelial stem cells, and intestinal stem cells [11-16]. BMI1 is also involved in the tumorigenesis of various cancer types [17].

PCGF6, also known as MBLR and RNF134, is a core component of a noncanonical PRC1 complex that is composed of some canonical components, such as RNF2, CBX3, CXB4, CXB6, CXB7, and CXB8, as well as some noncanonical components, such as L3MBTL2, E2F6, WDR5, HDAC1, and RYBP $[4,18]$. The RING finger domain of PCGF6 is located in the central region and exhibits high similarity to the analogous domain of PCGF2 and BMI1; it mediates the interaction between PCGF6 and RNF2, a core component of PRC1 with E3 ligase activity for histone H2A monoubiquitination at lysine 119 (H2AK119u1) [19]. Surprisingly, PCGF6 can directly interact with JARID1d/KDM5D, a trimethyl H3K4 demethylase, and regulate its enzymatic activity [20]. In addition, PCGF6 also can interact with BMI1[19]. However, so far we only know that PCGF6 possesses the transcriptional repression activity that is common to all other PcG proteins [19], the biological functions and related molecular mechanisms of PCGF6 are still unknown.

A previous study revealed that PCGF6 is expressed in multiple adult tissues of human and mouse at different levels, including liver, skeletal muscle, ovary, testis, thymus, heart, and pancreas. It is worth noting that PCGF6 is preferentially expressed in testis, and transcript and protein levels of PCGF6 in testis were significantly higher than in other tissues [19]. Additionally, the mRNA level of Pcgf6 in testicular biopsies with normal spermatogenesis is significantly higher than in testicular biopsies with round spermatid maturation arrest. Collectively, these results imply that PCGF6 may play critical roles in mammalian male germ cell development.

In this study, we systematically examined the expression pattern of PCGF6 in testis tissue at different developmental stages and the cellular localization in adult testis for the first time. Then, we investigated the effects of PCGF6 knockdown on in vitro proliferation and differentiation of GC-2 spd cells, an immortalized mouse spermatogenic cell line. To explore the underlying molecular mechanisms of PCGF6 in male germ cell development, we obtained the interaction partners of PCGF6 by co-immunoprecipitation. Additionally, we revealed that PLZF and OVOL1, two key transcription factors involved in spermatogenesis, could modulate Pcgf6 promoter activity. Taken together, this study is the first to reveal the biological role of 
PCGF6, and the results indicated that PCGF6 plays an important role in mammalian male germ cell development.

\section{Materials and Methods}

\section{Animals and cell line}

Male C57BL/6 mice used in this study were purchased from SLAC Laboratory Animal Co., Shanghai, China. All procedures involving animals were approved by the Institutional Animal Care and Use Committee at Shanghai Jiao Tong University, Shanghai, China [SYXK (Shanghai 2007-0025)], and were conducted in accordance with the National Research Council Guide for Care and Use of Laboratory Animals. HEK 293T/17 cells (CRL-11268) and GC-2 spd cells (CRL-2053), a mouse-derived spermatogenic line, were purchased from American Type Culture Collection.

\section{RNA isolation, reverse transcription, and quantitative real-time PCR}

Total RNA derived from mouse testes and cells was purified using Trizol Reagent (Invitrogen, UAS) and treated with DNase I (Fermentas, China) according to the instruction of the manufacturer. Total RNA was reverse transcribed into cDNA with oligo dT primers using the PrimeScript RT-PCR Kit (TaKaRa, Japan). Quantitative real-time PCR (qRT-PCR) was performed using FastStart Universal SYBR Green Master (Roche, China) on an ABI PRISM 7500 Sequence Detection System (Applied Biosystems) according to the procedure used by Sun et al. [21]. The relative expression of genes was calculated based on the $2^{-\Delta \Delta \mathrm{ct}}$ method using the GAPDH gene as an endogenous control. The primers used for qRT-PCR are shown in Table 1.

\section{Western blotting}

The testes and harvested cells were lysed with sodium dodecyl sulfate (SDS) cell lysis buffer (Beyotime, China) containing $1 \mathrm{mM}$ phenylmethanesulfonyl fluoride (PMSF). Then, samples (50 $\mu \mathrm{g}$ protein/lane) were electrophoresed by SDS polyacrylamide gel electrophoresis (PAGE) and transferred onto a nitrocellulose membrane. The membrane was blocked with $5 \%(\mathrm{w} / \mathrm{v})$ non-fat dry milk for $1 \mathrm{~h}$ at room temperature and subsequently incubated overnight at $4{ }^{\circ} \mathrm{C}$ with rabbit-anti-PCGF6 (1:500; Abcam, USA), goat-anti-PCGF6 (1:600; Santa Cruz, USA), rabbit-anti-HSPA2 (1:600; Proteintech, USA), or mouse-anti- $\beta$-tubulin $(1: 1000$; Santa Cruz, USA). Then, the membrane was incubated with a horseradish peroxidase-conjugated goat-antirabbit IgG (1:2000; Proteintech, USA), donkey-anti-rabbit (1:5000; Proteintech, USA), or goat-anti-mouse (1:5000; Proteintech, USA) for $1 \mathrm{~h}$. Chemiluminescence was detected using the ECL blot detection system (Amersham, USA).

\section{Fluorescent immunohistochemistry (IHC)}

Adult mouse testes were fixed with $4 \%$ paraformaldehyde at $4^{\circ} \mathrm{C}$ overnight and then were embedded in paraffin and sectioned. Rehydrated sections were incubated in $0.05 \%$ trypsin in phosphate-buffered saline (PBS) for $30 \mathrm{~min}$ at $37^{\circ} \mathrm{C}$, washed twice in PBS (10 min each), incubated for $10 \mathrm{~min}$ in blocking solution ( $10 \%$ normal goat serum in PBS) at room temperature, incubated with rabbit-anti-PCGF6 (1:50; Abcam, USA) overnight at $4^{\circ} \mathrm{C}$, incubated for $30 \mathrm{~min}$ at $37^{\circ} \mathrm{C}$ with goat anti-rabbit IgG-FITC (1:100; Proteintech, USA), washed twice in PBS (10 min each), and incubated at $37^{\circ} \mathrm{C}$ for $20 \mathrm{~min}$ with $4^{\prime}$, 6-diamidino-2-phenylindole (DAPI; 1:10,000) for nuclear staining. Coverslips were added to slides for observation. Fluorescent images were taken using a fluorescence microscope (Olympus IX51, Japan) with a digital camera (Olympus DP72, Japan).

Cell culture and establishment of a stable PCGF6-knockdown GC-2 spd cell line

HEK 293T cells and GC-2 spd cells were cultured in Dulbecco's modified Eagle medium containing $10 \%(\mathrm{v} / \mathrm{v})$ fetal bovine serum (GIBCO) at $37^{\circ} \mathrm{C}$ with $5 \% \mathrm{CO}_{2}$. Differentiation in vitro of GC-2 spd cells was induced by decreasing the temperature to $32^{\circ} \mathrm{C}$ [22]. We established a stable PCGF6-knockdown GC-2 spd cell line using the lentiviral shRNA expression system. Briefly, shRNA oligonucleotides specific to the PCGF6 open reading frame (ORF) (shRNA-449: AGGTTACCTGATAGATGCA) and a negative control (shRNA-NC: ACTACCGTTGTTATAGGTG) were designed and synthesized according to the user manual of the Lenti-X shRNA Expression System (Clontech, USA) and were inserted into the modified pLVX-shRNA2 
Sun et al.: PCGF6 Modulates Male Germ Cell Differentiation in Vitro

Table 1. List of the qRT-PCR primers used in this study

\begin{tabular}{|c|c|c|c|}
\hline \multirow[t]{2}{*}{ Gene Symbol } & \multicolumn{2}{|c|}{ Sequence $\left(5^{\prime}-3^{\prime}\right)$} & \multirow{2}{*}{$\begin{array}{l}\text { Product size } \\
\text { (bp) }\end{array}$} \\
\hline & Forward & Reverse & \\
\hline PCGF6 & GTACCAAACTCTACGGGAAATCC & CTCTTAACAGCGGAAAGTAGTCGT & 220 \\
\hline Ccna1 & GACCGTTCCAACCACCAACCAAT & CACTCAGGCAAGGCACAATCTCA & 247 \\
\hline Ccna2 & TGGACTGGCTGGTTGAGGTGG & TGCTTCTTGGAATAGGTATCGTCTG & 226 \\
\hline Cond1 & CAGAAGTGCGAAGAGGAGGTC & TCATCTTAGAGGCCACGAACAT & 130 \\
\hline Ccnd2 & CAGGATGATGAAGTGAACAC & GAGACAATCCACATCAGTG & 84 \\
\hline Ccnd3 & TTGCATCTATACGGACCAG & AGGTGTAATCTGTAGCACAG & 212 \\
\hline Ccne1 & AGGCCCTTAAGTGGCGTCTAA & CATAGGCCACTTGGACATAGACAT & 72 \\
\hline Ccne2 & GCTGCTGCCGCCTTATGTC & GCACCATCCAGTCTACACATTCC & 100 \\
\hline Cdk2 & CAAGTGGGCTCGGCAAGATTTTA & GCATCAGAGCCGAAGGTGGG & 178 \\
\hline Cdk3 & TTGTGTTGACTTTTGTGGGTTTC & CTGTGAGAGAGGTGCTATGGGG & 106 \\
\hline Cdk4 & GCCGACCAGTTGGGGAAAATCT & TCTTCTCACTCTGCGTCGCTTTC & 260 \\
\hline Cdk6 & ATTCCAAATCTGCTCAACCCATC & GGTTGGATGGCAGGTGAGAGTTC & 189 \\
\hline p15 & ATCCCAACGCCCTGAACCG & GCСCTGCTCTTCAGCCAAGTCTA & 257 \\
\hline p16 & CAAGAGCGGGGACATCAAGAC & GCTAAGAAGAAAAAGGCGGGCT & 209 \\
\hline p18 & TCGTCAGGACCCTAAAGAATGGC & GAAGCCTCCTGGCAATCTCCG & 187 \\
\hline p19 & TCTGGAAGAAGTCTGCGTCGG & CAGGGCATTGACATCAGCACC & 304 \\
\hline p21 & TCTTGCACTCTGGTGTCTGAG & ATCTGCGCTTGGAGTGATAG & 115 \\
\hline p27 & CCCAGACCTGCGCGCTACTG & GACCACCGCCTCGCCTCTCT & 182 \\
\hline p57 & AGAGAACTGCGCAGGAGAAC & TCTGGCCGTTAGCCTCTAAA & 141 \\
\hline Acr & CCTCCCAGTACAGTGGACCAAG & TTTTCTCAGGAATGGAGGAAGG & 100 \\
\hline Pwil2 & TGACCTGTGCATCCCCTTCT & TCCCCACAAGCTTCATATCCA & 74 \\
\hline Pgk2 & CTGGTTGGATGGGCTTGGAC & GGTGACACAGCCATTGGAGG & 182 \\
\hline Prm1 & $\begin{array}{l}\text { AGGTGTAAAAAATACTAGATGC } \\
\text { ACAGAATAG }\end{array}$ & TTCAAGATGTGGCGAGATGCT & 97 \\
\hline Tex14 & GATGAGCTTCCACCACCAGC & GTTCTCATGTGACGCCAGGG & 90 \\
\hline Tnp2 & GCTCAGGGCGAAGATACAAGTG & TGTGACATCATCCCAACAGTCC & 103 \\
\hline GAPDH & GTCGTGGAGTCTACTGGTGTC & GAGCCCTTCCACAATGCCAAA & 240 \\
\hline
\end{tabular}

vector containing the neomycin resistance gene using BamHI/EcoRI cloning sites. Subsequently, shRNAexpressing lentiviral plasmid was cotransfected with plasmids pLP1, pLP2, and pLP/VSVG into HEK 293T cells to produce lentiviral particles using GenJet Plus DNA In Vitro Transfection Reagent (SignaGen, USA) according to the user manual of the ViraPower Lentiviral Expression System (Invitrogen, USA). GC-2 spd cells were cultured in medium containing recombinant lentiviral particles and $5 \mu \mathrm{g} / \mathrm{mL}$ polybrene for $48 \mathrm{~h}$ and then selected with $3 \mathrm{mg} / \mathrm{mL}$ geneticin for 3 weeks [22].

\section{Cell proliferation assay}

The effect of PCGF6 knockdown on GC-2 spd cell proliferation was tested by performing a WST-8 assay and 5-ethynyl-20-deoxyuridine (EdU) incorporation assay using the CCK-8 cell proliferation kit (Dojindo, Japan) and EdU assay kit (Ribobio, China), respectively. All experiments were done in triplicate, and three independent experiments were performed.

For the CCK-8 assay, cells were seeded into a 96-well plate at $1 \times 10^{3}$ cells per well with $100 \mu \mathrm{L}$ complete medium and cultured at $37^{\circ} \mathrm{C} ; 10 \mu \mathrm{L}$ CCK- 8 solution was added to each well after $24 \mathrm{~h}, 48 \mathrm{~h}, 72 \mathrm{~h}$, and $96 \mathrm{~h}$. Plates were incubated at $37^{\circ} \mathrm{C}$ for $2 \mathrm{~h}$, and then the absorbance at $450 \mathrm{~nm}$ was measured with an Enspire 2300 Multilabel Reader (PerkinElmer, USA).

For the EdU incorporation assay, cells $\left(1 \times 10^{5}\right)$ were cultured in 24 -well plates and exposed to 20 $\mu \mathrm{M}$ EdU for $2 \mathrm{~h}$ at $37^{\circ} \mathrm{C}$. The cells were then fixed in $4 \%$ formaldehyde for $30 \mathrm{~min}$ at room temperature and permeabilized in $0.5 \%$ Triton X-100 for 10 min. Cells were washed with PBS, and each well was incubated with $400 \mu \mathrm{L} 1 \times$ Apollo $^{\circledR}$ reaction cocktail for $30 \mathrm{~min}$ at room temperature. DNA was then stained with $5 \mu \mathrm{g} /$ mL Hoechst 33342 (200 $\mu \mathrm{L}$ per well) for $10 \mathrm{~min}$ and imaged under a fluorescence microscope. The EdUpositive cells (green cells) were counted using Image-Pro Plus 6.0 software. The EdU incorporation rate was expressed as the ratio of EdU-positive cells to total Hoechst 33342-positive cells (blue cells).

Flow cytometry

For the cell cycle assay, cells were harvested and fixed with $70 \%$ alcohol in PBS overnight at $4{ }^{\circ} \mathrm{C}$. After washing in PBS, cells were treated with propidium iodide (PI) staining solution containing $50 \mu \mathrm{g} / \mathrm{mL} \mathrm{PI}, 100$ $\mu \mathrm{g} / \mathrm{mL}$ RNase, and $0.2 \%(\mathrm{v} / \mathrm{v})$ Triton X-100 at room temperature for $30 \mathrm{~min}$ in the dark. The percentage of 
cells in different phases of the cell cycle was determined by a flow cytometer (BD Biosciences, USA) with the ModFit program.

For haploid cell population analysis, harvested cells were stained with freshly prepared PBS containing a final concentration of $10 \mu \mathrm{g} / \mathrm{mL}$ Hoechst 33342 for $30 \mathrm{~min}$ at $37^{\circ} \mathrm{C}$ in the dark. The cell suspension was analyzed by a flow cytometer. Gating parameters of haploid cells $(1 \mathrm{~N})$ were set using sperm cells isolated from the mouse epididymis. The percentage of haploid cells was determined with the $1 \mathrm{~N}$ gating parameters.

\section{Co-immunoprecipitation}

Protein from testes was extracted using radio-immunoprecipitation assay (RIPA) lysis buffer (Beyotime, China). The cell lysate was first pre-cleared by adding protein A/G-agarose beads (Abmart, China), incubating at $4^{\circ} \mathrm{C}$ for $10 \mathrm{~min}$, and centrifugation. The supernatant was then incubated with rabbitanti-PCGF6 (Abcam, USA) or normal rabbit IgG isotype control antibody (Abmart, China) for $2 \mathrm{~h}$ at $4^{\circ} \mathrm{C}$, which was followed by the addition of protein A/G-agarose and overnight incubation. The immunoprecipitate was collected by centrifugation and washed with RIPA lysis buffer. The eluting complex was subjected to SDSPAGE separation and Coomassie brilliant blue staining for mass spectrometry or western blotting. Mass spectrometry was performed by Sangong Biotech, Shanghai, China.

\section{Promoter activity analysis}

The promoter regions $(2,000 \mathrm{bp}$ region upstream of the ATG codon) of PCGF6 was amplified from mouse genomic DNA and cloned upstream of firefly luciferase in the pGL3-Basic vector (Promega, USA). DNA sequences containing the ORF of PLZF and OVOL1 were amplified from adult mouse testis cDNA and cloned into the pcDNA $3.1(+)$ vector to overexpress PLZF and OVOL1. Twenty-four hours before transfection, HEK $293 \mathrm{~T}$ cells were seeded in 24 -well plates at $1 \times 10^{5}$ cells/well. Each well of cells was transfected with 500 ng pGL3-Basic (as the no promoter control) or luciferase reporter plasmids (pGL-PCGF6) containing the promoter region of PCGF6. In co-transfection experiments, 500 ng PLZF or OVOL1 expression vector was used. Each well of cells was simultaneously transfected with $50 \mathrm{ng}$ Renilla luciferase reporter plasmid (phRLTK) as a control for transfection efficiency. The promoter activity of each group was determined by the DualLuciferase Reporter Assay System (Promega, USA) $24 \mathrm{~h}$ after transfection according to the technical manual.

\section{Statistical analysis}

All experiments were repeated three times. The results are presented as means \pm SD and were analyzed by analysis of variance and Student's $t$-test using the statistical software SPSS 20.0. Differences were considered significant when the $P$ value was $<0.05$.

\section{Results}

\section{PCGF6 is highly expressed in testes of 18-day-old mice}

To investigate the role of PCGF6 in male germ cell development, we first examined the expression profile of PCGF6 in testes from 6-, 12-, 18-, 24-, and 30-day-old and 8-weekold mice by qRT-PCR and western blot analysis. Results revealed that PCGF6 was weakly expressed in testes of 6- and 12-day-old mice, and its expression level was sharply increased on 18 days and maintained a higher level during later developmental stages both at the mRNA level (Fig. 1) and protein level (Fig. 1B, C). According to the differentiation time course of the first wave of spermatogenesis in mouse, the expression pattern of PCGF6 hinted that it might play an essential role during the pachytene stage of meiosis and the post-meiotic stages of spermatogenesis.

PCGF6 protein is predominantly localized in pachytene spermatocytes and elongated spermatids

To obtain the cellular localization of PCGF6 protein in mouse testes, we used a PCGF6specific antibody to perform immunolocalization analysis in testes from adult mouse (8 weeks old). Fluorescent IHC staining revealed that PCGF6 protein could be detected in all types of male germ cells including spermatogonia, pachytene spermatocytes, round 


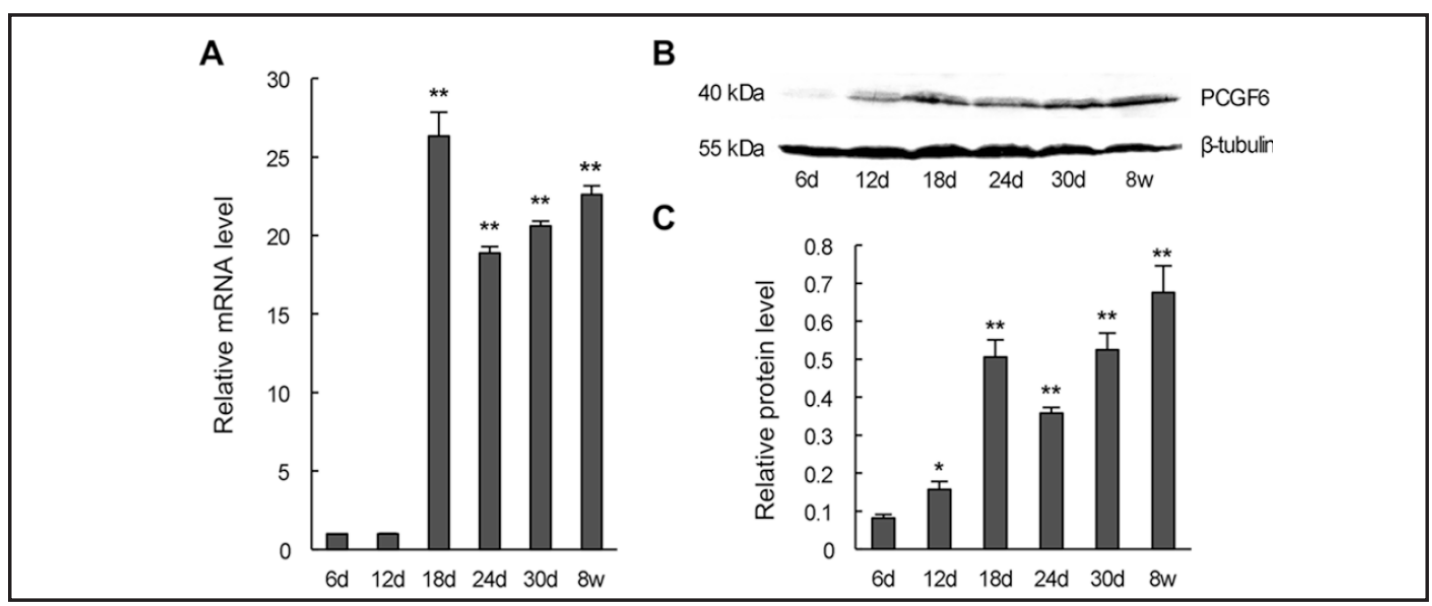

Fig. 1. Expression pattern of PCGF6 in mouse testis at different postnatal stages. A: The relative mRNA level of PCGF6 was determined by qRT-PCR. B: The protein level of PCGF6 was shown by western blotting. C: Quantitative western blot results. The results were expressed as the ratio of PCGF6 to $\beta$-tubulin. The horizontal axis indicates the six time points of postnatal testis development from 6 days (d) to 8 weeks (w) after birth. The bars represent the means \pm SD from three independent experiments. ${ }^{*} P<0.05,{ }^{*} P<0.01$ by the Student's $t$-test.

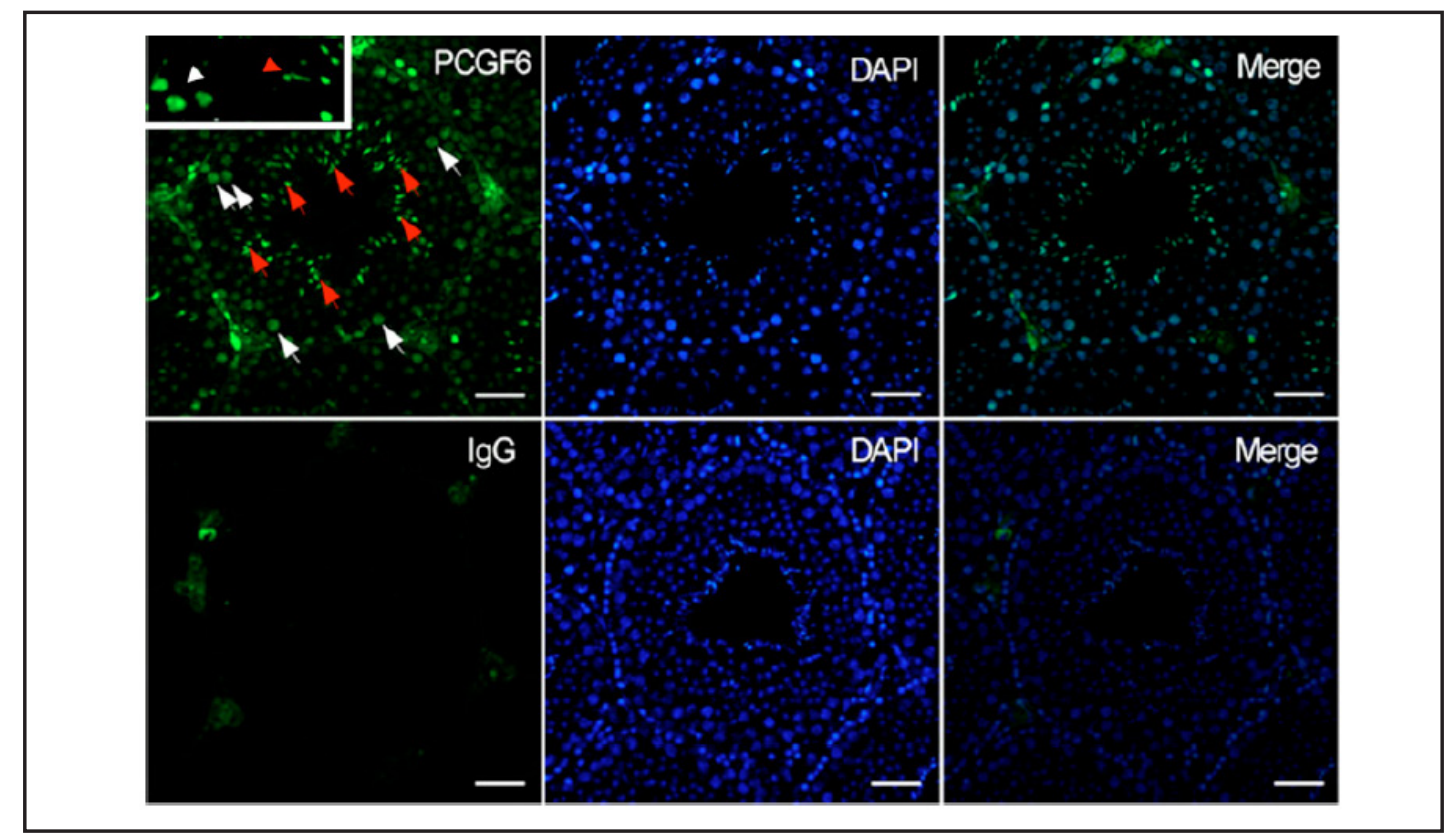

Fig. 2. Immunohistochemical localization of PCGF6 in adult mouse testis. Upper row: Sections were incubated with rabbit-anti-PCGF6, DAPI, and their merged image. Lower row: Sections were incubated with rabbit normal IgG (negative control), DAPI, and their merged image. White arrows indicate positive pachytene spermatocytes; red arrows indicate positive elongated spermatids. Scale bar, $40 \mu \mathrm{m}$.

spermatids, and elongated spermatids. Notably, immunofluorescence signals were much stronger in pachytene spermatocytes and elongated spermatids than in other types of germ cells (Fig. 2). This result was consistent with the expression pattern of PCGF6 in mouse testis at different stages of postnatal development and further suggested that PCGF6 was likely to participate in meiosis and post-meiotic development of male germ cells. 


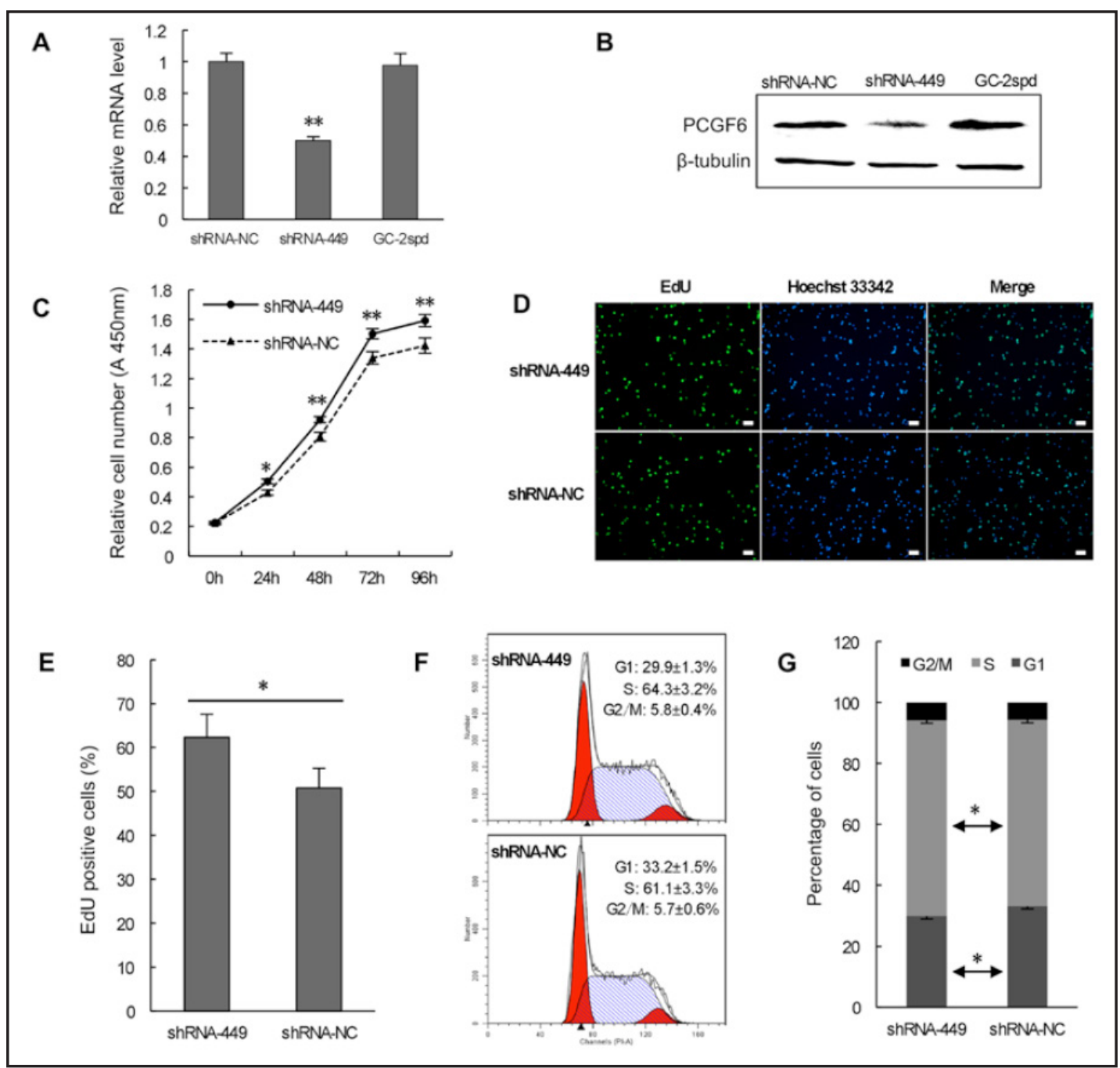

Fig. 3. Effects of PCGF6 knockdown on GC-2 spd cell proliferation. A: The relative mRNA level of PCGF6 in PCGF6-knockdown and control GC-2 spd cells was determined by qRT-PCR. B: The protein level of PCGF6 in PCGF6-knockdown and control GC-2 spd cells was determined by western blotting. C: Analysis of cell proliferation using the CCK-8 assay. D: Analysis of cell proliferation using EdU labeling. Scale bar, $50 \mu \mathrm{m}$. E: The percentage of EdU-positive cells. F: The cell cycle distribution of stable PCGF6-knockdown and control GC-2 spd cells is shown by flow cytometric histograms. G: The percentage of cells in three phases of the cell cycle is shown by a stacked column chart. ${ }^{*} P<0.05,{ }^{* *} P<0.01$ by the Student's $t$-test.

\section{PCGF6 knockdown accelerates proliferation of GC-2 spd cells}

Previous studies have found that some PCGF homologs, such as PCGF1/NSPc1, PCGF2/ Mel-18, and PCGF4/Bmi1, could regulate cell proliferation and differentiation. To explore whether PCGF6 is involved in the proliferation of male germ cells, we determined the effects of stable PCGF6 knockdown on the proliferation of GC-2 spd cells, an immortalized mouse spermatocyte cell line. Here, we stably silenced PCGF6 in GC-2 spd cells using recombinant lentiviral particles containing shRNAs targeted to PCGF6. These cells exhibited significantly reduced expression of PCGF6 at both the mRNA (Fig. 3A) and protein levels (Fig. 3B) compared to control lentiviral vector-infected cells. The effect of PCGF6 silencing on proliferation was measured using the CCK-8 and EdU incorporation assays. As shown in Fig. 3C, stable silencing of PCGF6 significantly increased the relative cell number. As anticipated, the number of EdU-positive cells was approximately 11\% higher in PCGF6-knockdown cells than in control cells (Fig. 3D, E). These results indicated that PCGF6 knockdown promoted 
Fig. 4. Effects of PCGF6 knockdown on the mRNA expression levels of cell cycle regulatory genes in GC-2 spd cells. A: The relative mRNA levels of cyclin genes. B: The relative mRNA level of CDK genes. C: The relative mRNA level of CDKI genes. The relative mRNA levels were determined by qRTPCR and were normalized to the mRNA levels of the GAPDH gene. Data represent the mean $\pm \mathrm{SD}$ from three independent experiments. ${ }^{*} P<0.05,{ }^{* *} P<0.01$ by the Student's $t$-test.

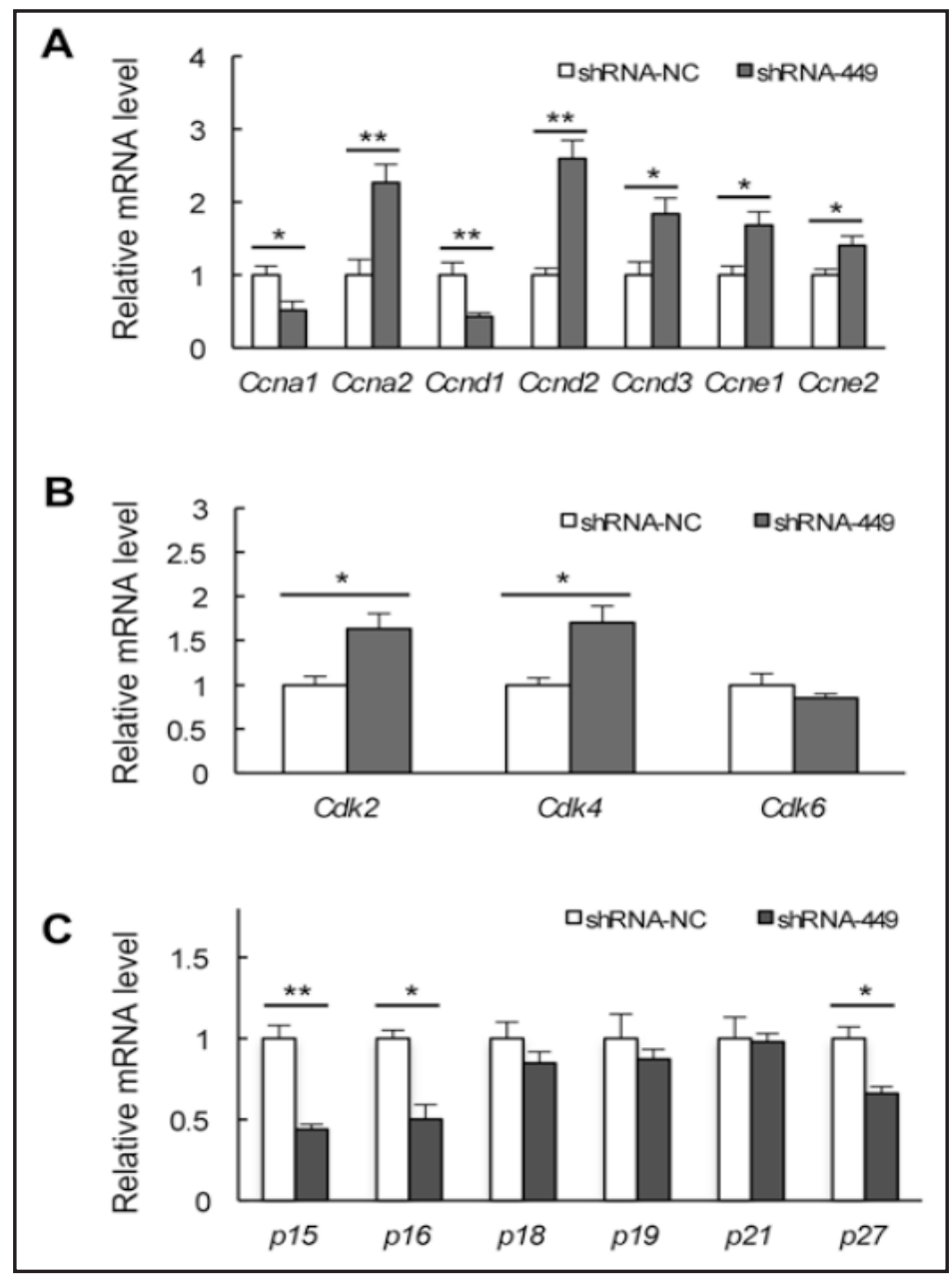

the proliferation of GC-2 spd cells. Flow cytometry analysis revealed that PCGF6 knockdown resulted in a significantly lower number of cells in the G1 phase $(P<0.05)$ and a significantly higher number of cells in the $S$ phase $(P<0.05)$ compared with the negative control (Fig. 3F, $G)$. This result suggested that the knockdown of PCGF6 could promote the transition of cells from G1 phase to $\mathrm{S}$ phase. To investigate the potential mechanisms underlying the ability of PCGF6 to influence GC-2 spd cell proliferation, we examined the relative mRNA levels of some cell cycle regulator genes related to the G1/S transition by qRT-PCR. We found that PCGF6 knockdown upregulated five cyclin genes (Ccna2, Ccnd2, Ccnd3, Ccne1, and Ccne2) (Fig. 4A) and two cyclin-dependent kinase (CDK) genes (Cdk2 and Cdk4) (Fig. 4B), while it downregulated three CDK-inhibitor (CDKI) genes ( $p 15, p 16$, and $p 27)$ (Fig. 4C).

\section{PCGF6 knockdown suppresses the differentiation of GC-2 spd cells in vitro}

The expression pattern of PCGF6 in testis tissue suggested that PCGF6 was most likely to be involved in male meiosis. To explore the role of PCGF6 in meiosis, we investigated the effects of PCGF6 knockdown on GC-2 spd cell differentiation in vitro based on the following characteristic of GC-2 spd cells: a small portion of cells can undergo meiosis to produce a haploid population and express some post-meiotic marker genes following low temperature culture $\left(32^{\circ} \mathrm{C}\right)$ for several weeks $[23,24]$. Stable PCGF6-knockdown and control GC-2 spd cell lines were cultured at $32^{\circ} \mathrm{C}$ for 3 weeks, and the haploid cell population was analyzed by flow cytometry. As shown in Fig. 5, PCGF6 knockdown resulted in a significantly lower portion of haploid cells in the GC-2 spd cell population $(P<0.05)$ than in the negative control. These results indicated that PCGF6 knockdown could hinder the differentiation of GC-2 spd cells in vitro. In addition, we found that some haploid spermatid marker genes were significantly 
A

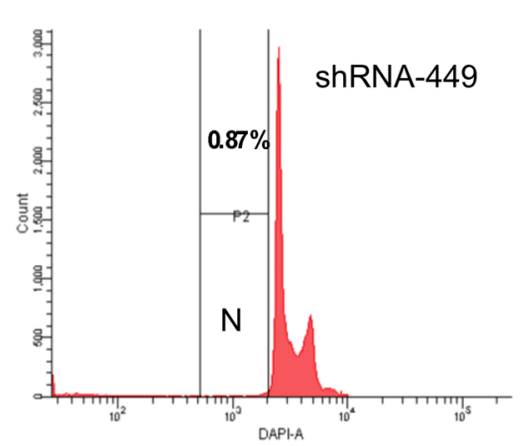

C

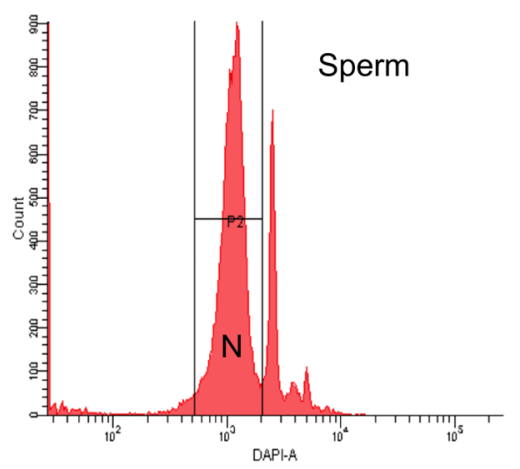

B

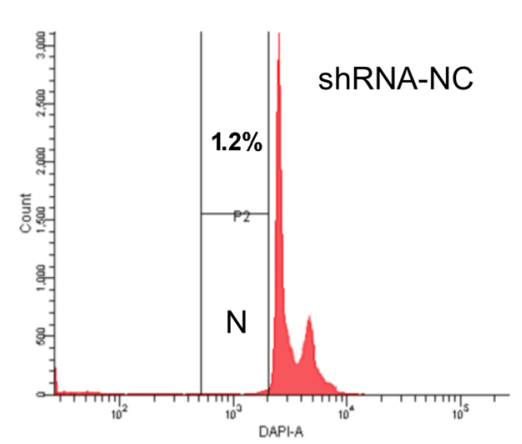

D

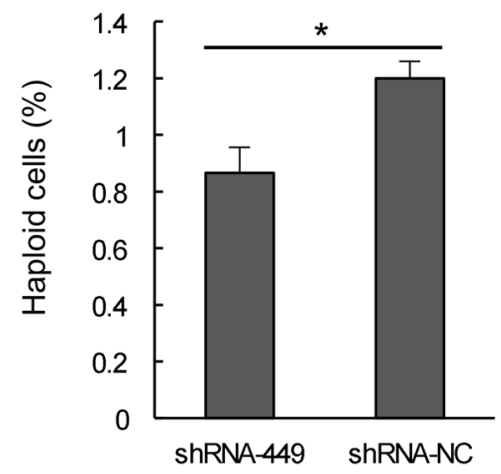

Fig. 5. Determination of the percentage of haploid cells in stable PCGF6-knockdown and control GC-2 spd cells cultured at $32^{\circ} \mathrm{C}$ for 3 weeks by flow cytometry. A: The percentage of haploid cells in the stable PCGF6 knockdown GC-2 spd cell line was determined by flow cytometry. B: The percentage of haploid cells in the negative control GC-2 spd cell line was determined by flow cytometry. C: Flow cytometry parameters of haploid cells $(1 \mathrm{~N})$ were established using mouse sperm cells. D: The percentage of haploid cells is shown by a histogram. Data represent the mean \pm SD from three independent experiments. ${ }^{*} P<0.05$ by the Student's t-test.

Fig. 6. Effects of PCGF6 knockdown on the mRNA expression level of haploid spermatid marker genes in the GC-2 spd cell line. The relative mRNA levels were determined by qRT-PCR and were normalized to the mRNA levels of the $G A P D H$ gene. Data represent the mean $\pm \mathrm{SD}$ from three independent experiments. ${ }^{*} P<0.05$, ${ }^{*} P<0.01$ by the Student's $t$-test.

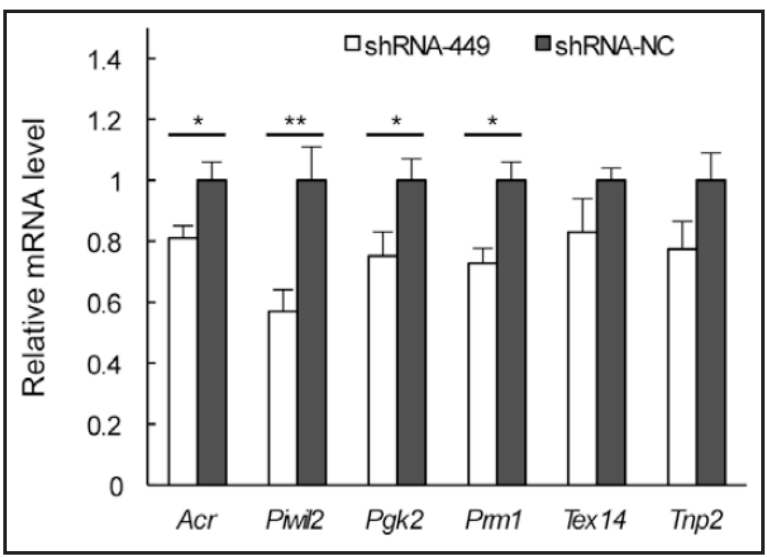

downregulated in PCGF6-knockdown cell lines, including Acr, Piwil2, Pgk2, and Prm1 $(P<$ 0.05 ) (Fig. 6). This result indirectly indicated that silencing PCGF6 expression suppressed the differentiation of GC-2 spd cells.

PCGF6 protein interacts with heat shock-related 70-kDa protein 2 (HSPA2)

We know that components of PRC1 are complicated and variable. To unveil the potential interaction partners of PCGF6 in testis tissue, we performed immunoprecipitation on total protein derived from adult mouse testes with rabbit-anti- PCGF6 and normal rabbit 


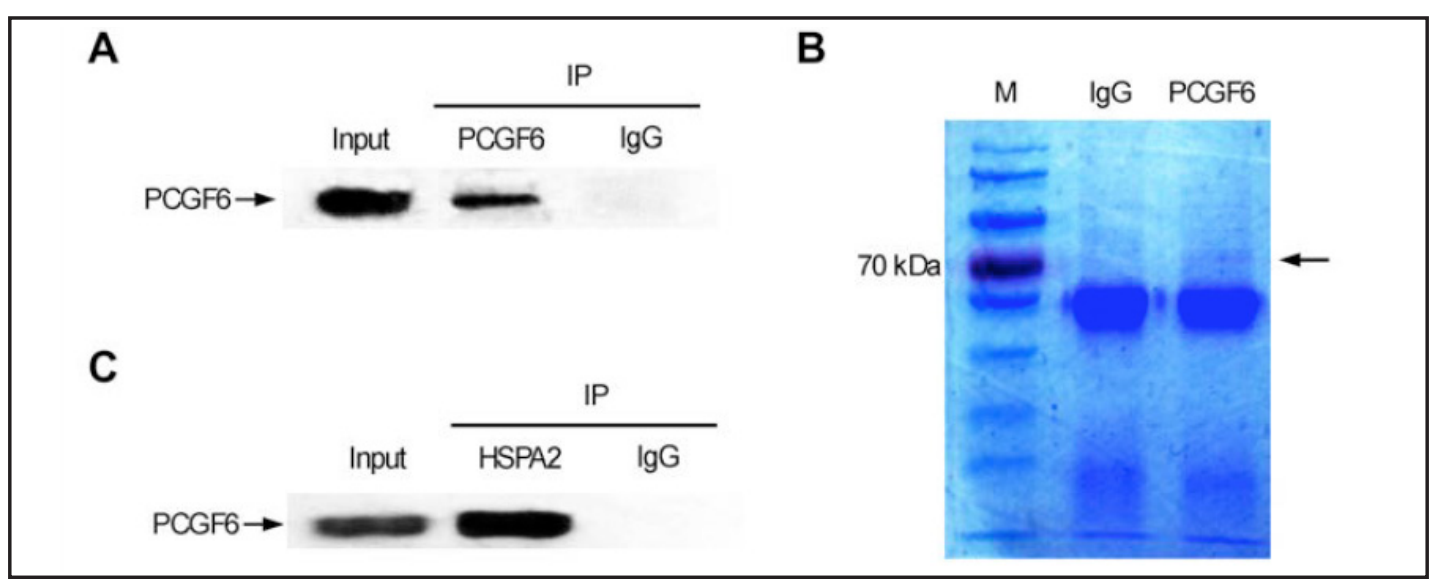

Fig. 7. PCGF6 co-immunoprecipitated with HSPA2 from adult mouse testis extracts. A: Identification of immunoprecipitated PCGF6 by western blotting. Testis lysates were subjected to immunoprecipitation with rabbit-anti-PCGF6 (PCGF6) or rabbit control IgG antibodies (IgG). The input (testis lysate) and immunoprecipitation (IP) fractions with goat-anti-PCGF6 were also subjected to western blot. B: An SDS-PAGE gel stained with Coomassie brilliant blue after co-immunoprecipitation. Testis lysates were subjected to immunoprecipitation with rabbit-anti-PCGF6 (PCGF6) or control IgG antibodies (IgG). M: Protein marker (SM0671, Thermo Scientific). The arrow indicates the differential band. C: Identification of PCGF6 co-immunoprecipitated with HSPA2 by western blot. Testis lysates were subjected to immunoprecipitation with rabbit-anti-HSPA2 (HSPA2) or rabbit control IgG antibodies (IgG). The input (testis lysate) and immunoprecipitation (IP) fractions with goat-anti-PCGF6 were also subjected to western blot.

Table 2. Potential binding sites for OVOL1 and PLZF in mouse PCGF6 gene promoter

\begin{tabular}{cccc}
\hline Transcription factor & Start position & End position & Strand \\
\hline OVOL1 & 858 & 872 & + \\
PLZF & 330 & 344 & - \\
PLZF & 654 & 668 & - \\
PLZF & 977 & 991 & - \\
PLZF & 1679 & 1693 & + \\
PLZF & 1723 & 1737 & - \\
\hline
\end{tabular}

IgG isotype control antibody. Western blotting with goat-anti-PCGF6 confirmed that the immunoprecipitation showed high specificity (Fig. 7A). The eluting immunoprecipitate was separated by SDS-PAGE and stained with coomassie brilliant blue, and an obvious differential band with the size around $70 \mathrm{kDa}$ was observed in the anti-PCGF6 lane but not in the control IgG lane (Fig. 7B). This differential band was identified as heat shock-related 70-kDa protein 2 (HSPA2) by mass spectrometry (data not shown). To confirm the result obtained from mass spectrometry, we performed immunoprecipitation with rabbit-anti-HSPA2 and western blot with goat-anti-PCGF6. As shown in Fig. 7C, PCGF6 could be specifically immunoprecipitated by anti-HSPA2 antibody and co-immunoprecipitated with HSPA2.

The promoter activity of PCGF6 can be enhanced and inhibited by the overexpression of OVOL1 and PLZF, respectively

Expression pattern of PCGF6 suggested that its transcriptional activity was tightly regulated during male germ cell development. To explore the mechanism of the transcriptional regulation of $P C G F 6$, potential transcription factor binding sites in a putative PCGF6 promoter region (2,000-bp region upstream of the ATG codon) was predicted using MatInspector software (Genomatix, Germany) [25]. As shown in Table 2, one homologlike 1 (OVOL1) and five promyelocytic leukemia zinc finger (PLZF) binding sites existed in 
Fig. 8. Effects of the overexpression of OVOL1 and PLZF on the transcriptional activity of the mouse PCGF6 promoter in HEK 293T cells. A: The transcriptional activation of the PCGF6 promoter is potentiated by OVOL1. B: The transcriptional repression of the PCGF6 promoter is potentiated by PLZF. The relative luciferase activity was determined by dual luciferase assays and normalized to the Renilla luciferase activity. Data represent the mean \pm SD from three independent experiments. ${ }^{*} P<0.05,{ }^{* *} P<0.01$ by the Student's $t$-test.

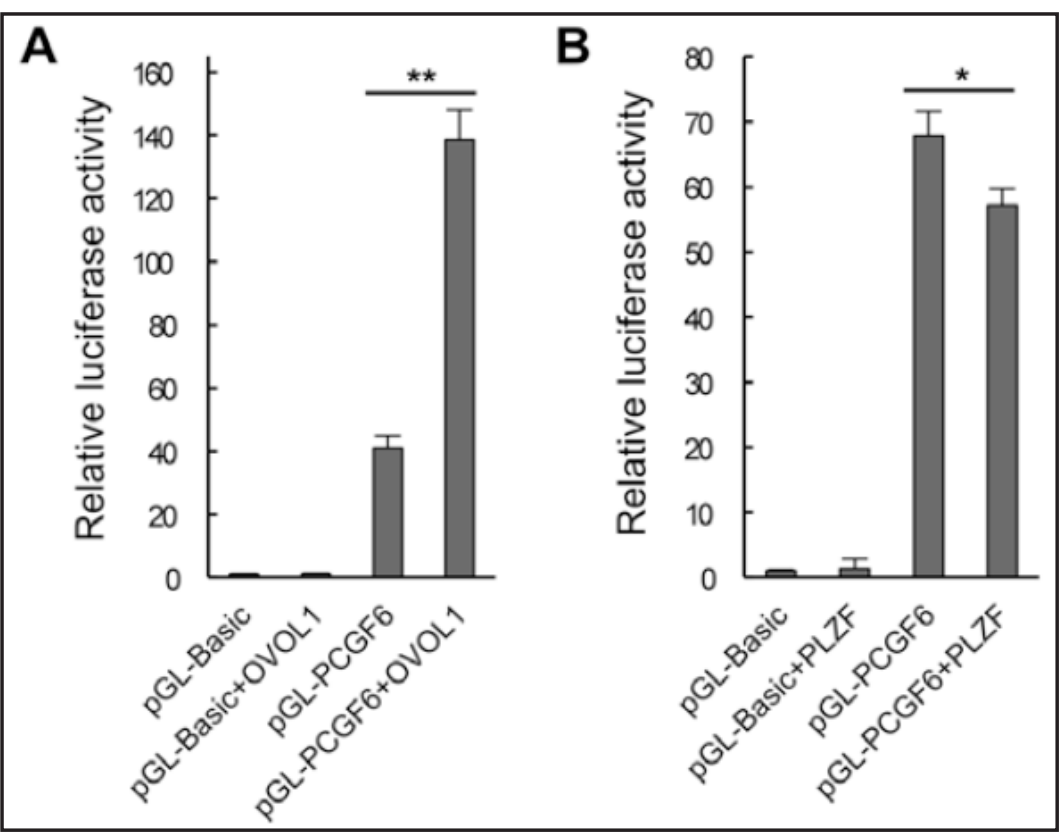

Fig. 9. Expression pattern of PCGF homologs in six mouse organs. Expression pattern was generated using RNA-sequencing data from David et al. [31]. The bar code on the right represents expression level: great color saturation means higher expression.

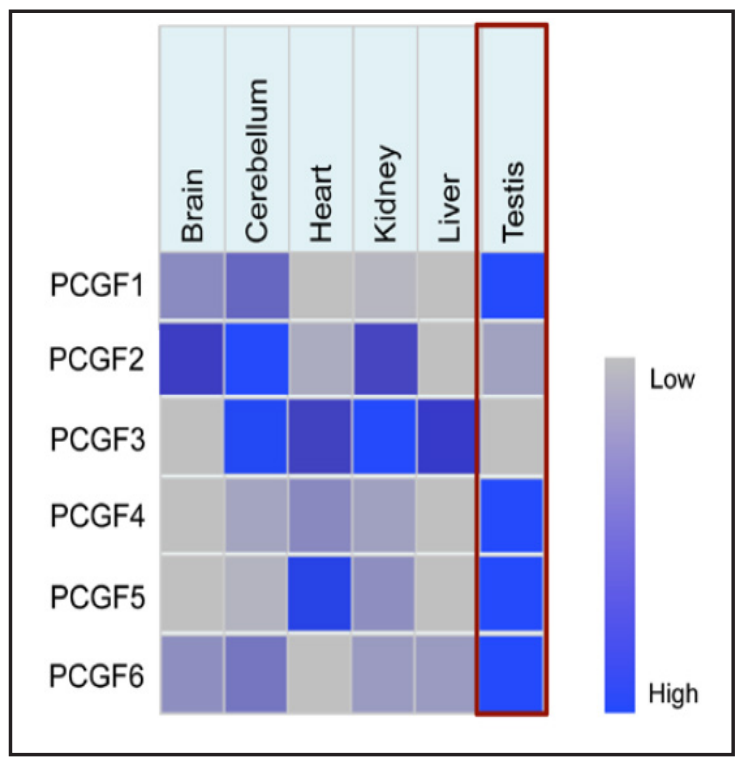

the PCGF6 promoter region. Previous studies have demonstrated that OVOL1 was highly expressed in pachytene spermatocytes and involved in meiotic pachytene progression as a key transcription factor [26]. In this study, we found that PCGF6 was also highly expressed in pachytene spermatocytes. Thus, we speculated that OVOL1 positively modulated the transcription of PCGF6 by binding to its promoter. PLZF, a well-known spermatogonial stem cell (SSC) marker, is directly involved in SSC self-renewal by negatively regulating SSC differentiation genes, such as c-Kit, TORC1, and RARs [27-29]. In this study, we found that PCGF6 showed a low expression level in spermatogonia; therefore, we speculated that the transcription of PCGF6 was suppressed by PLZF. To test our hypothesis, we performed PCGF6 promoter-driven luciferase reporter assays in HEK 293T cells transiently overexpressing OVOL1 and PLZF. As expected, we found that the luciferase activity was significantly increased in HEK 293T cells overexpressing OVOL1 compared with the control $(P<0.01)$ (Fig. 8A) and significantly decreased in HEK 293T cells overexpressing PLZF $(P<0.05)$ (Fig. $8 \mathrm{~B})$, indicating that the transcriptional activity of the PCGF6 promoter could be enhanced and inhibited by the overexpression of OVOL1 and PLZF, respectively. 
PCGF1, BMI1, and PCGF5 are also highly expressed in mouse testes like PCGF6

So far, we know that there are six distinct typess of PRC1 complexes that are identified by one of six distinct PCGF homologs (PCGF1, PCGF2, PCGF3, BMI1, PCGF5, and PCGF6) [4]. To understand the expression status of other PCGF paralogs in mouse testis, we obtained the expression pattern of six PCGF homologs in six mouse organs using RNA-sequencing data from David et al. by Expression Atlas of EMBL-EBI (http://www.ebi.ac.uk/gxa) [30]. We found that PCGF1, BMI1, and PCGF5 are also highly expressed in mouse testes (Fig. 9). Especially for PCGF1 and BMI1, their expression features in six different organs is very similar to PCGF6 (Fig. 9), implying that both of these PCGF homologs may play important roles in male germ development like PCGF6 and there maybe some functional redundancy between these factors.

\section{Discussion}

Previous studies have revealed that PCGF6, one of six PCGF homologs, can form a noncanonical PRC1 complex with some related partners and is preferentially expressed in testis tissue [19]. However, our understanding of the biological function of PCGF6 is still extremely poor. This study is the first systematic study of the biological function of PCGF6.

First, this paper shows that PCGF6 could negatively regulate cell proliferation. Previous research indicated that some PCGF homologs tend to positively regulate stem cell and tumor cell proliferation. For example, BMI1 is a well-known stem cell factor and oncogene that is extensively involved in stem cell and tumor cell proliferation by inhibiting several CDKI genes, such as $p 16$ and $p 19$, and activating Wnt, Hedgehog, and the TGF- $\beta$ /BMP pathway [3133]. Some PCGF homologs tend to be involved in cell differentiation by negatively regulating proliferation. For instance, PCGF2/Mel-18 can suppress cell proliferation and promote cell differentiation by negatively regulating several CDK genes, such as $C d k 2$ and $C d k 4$, as well as the Wnt and Akt pathway $[9,34]$. In this study, we showed that the knockdown of PCGF6 could accelerate GC-2 spd cell proliferation by promoting the G1/S transition and altering the expression of some cell cycle regulators. Remarkably, some cyclin and CDK genes that were upregulated upon PCGF6 knockdown are highly expressed in SSCs or involve in SSC self-renewal; these genes include Ccna2, Ccnd2, Ccnd3, and CDK4 [35, 36]. In addition, we identified some CDKI genes, such as $p 15, p 16$, and $p 27$, which were direct targets of the stem cell factor BMI1 and were downregulated upon PCGF6 knockdown. According to the above discussion, our results indicated that PCGF6 exhibited the ability to negatively regulate cell proliferation similar to PCGF2.

Second, this paper shows that PCGF6 participates in the differentiation of male germ cells by functioning in meiosis. In this study, PCGF6 showed a progressive increase in testes mRNA and protein until 18 days, when late pachytene spermatocytes are the most abundant population of germ cells in the seminiferous tubules and the meiotic divisions begin [37]. Consistent with this result, immunolocalization analysis showed that PCGF6 was highly expressed in pachytene spermatocytes. Expression characteristics suggested that PCGF6 was very likely to function in meiosis. Subsequently, we found that in vitro differentiation of the spermatogenic cell line GC-2 spd was inhibited by PCGF6 knockdown, indicating that PCGF6 could affect male germ cell differentiation by acting on meiosis. Importantly, we obtained a novel interaction partner of PCGF6, HSPA2. HSPA2, a testis-specific protein, is specifically expressed in meiotic and post-meiotic male germ cells. It has been found that HSPA2 plays critical roles in the completion of meiosis during male germ cell differentiation, and its knockout mice lack post-meiotic spermatids and mature sperm and was infertile [38]. Thus, the finding that PCGF6 indirectly interacts with HSPA2 further suggested that PCGF6 is involved in male meiosis. In addition, in this study, we found that the promoter activity of PCGF6 could be enhanced by OVOL1, a critical meiotic pachytene progression regulator of male germ cells, and this finding provides further evidence that PCGF6 is involved in male meiosis.

\section{KARGER}


In summary, this is the first systematic study to explore biological roles of PCGF6, a PcG protein that is highly expressed in testis tissue. Our results revealed that PCGF6 was predominantly expressed in meiotic and post-meiotic male germ cells and could modulate male germ cell proliferation and differentiation. We also found that PCGF6 could interact with HSPA2 and that its promoter activity could be affected by OVOL1and PLZF. The data presented in this paper provide a solid foundation for further elucidation of the significance of PCGF6 in male cell development.

\section{Acknowledgments}

This work was supported by National Basic Research Program of China (grant numbers 2013CB967401; http://www.most.gov.cn) and the National Nature Science Foundation of China (grant number 81370675, 81200472 and 81421061; http://www.nsfc.gov.cn)

\section{References}

1 Niessen HE, Demmers JA, Voncken JW: Talking to chromatin: Post-translational modulation of polycomb group function. Epigenetics Chromatin 2009;2:10.

2 Aloia L, Di Stefano B, Di Croce L: Polycomb complexes in stem cells and embryonic development. Development 2013;140:2525-2534.

3 Lanzuolo C, Orlando V: Memories from the polycomb group proteins. Annu Rev Genet 2012;46:561-589.

- 4 Gao Z, Zhang J, Bonasio R, Strino F, Sawai A, Parisi F, Kluger Y, Reinberg D: PCGF homologs, CBX proteins, and RYBP define functionally distinct PRC1 family complexes. Mol Cell 2012;45:344-356.

5 Schwartz YB, Pirrotta V: A new world of polycombs: Unexpected partnerships and emerging functions. Nat Rev Genet 2013;14:853-864.

-6 Chen T, Dent SY: Chromatin modifiers and remodellers: Regulators of cellular differentiation. Nat Rev Genet 2014;15:93-106.

7 Gong Y, Yue J, Wu X, Wang X, Wen J, Lu L, Peng X, Qiang B, Yuan J: NSPc1 is a cell growth regulator that acts as a transcriptional repressor of p21Waf1/Cip1 via the RARE element. Nucleic Acids Res 2006;34:61586169.

8 Ross K, Sedello AK, Todd GP, Paszkowski-Rogacz M, Bird AW, Ding L, Grinenko T, Behrens K, Hubner N, Mann M, Waskow C, Stocking C, Buchholz F: Polycomb group ring finger 1 cooperates with Runx1 in regulating differentiation and self-renewal of hematopoietic cells. Blood 2012;119:4152-4161.

-9 Lee J-Y, Jang K-S, Shin D-H, Oh M-Y, Kim H-J, Kim Y, Kong G: Mel-18 negatively regulates ink4a/arfindependent cell cycle progression via akt inactivation in breast cancer. Cancer Res 2008;68:4201-4209.

10 Kajiume T, Ninomiya Y, Ishihara H, Kanno R, Kanno M: Polycomb group gene Mel-18 modulates the selfrenewal activity and cell cycle status of hematopoietic stem cells. Exp Hematol 2004;32:571-578.

11 Molofsky AV, He S, Bydon M, Morrison SJ, Pardal R: Bmi-1 promotes neural stem cell self-renewal and neural development but not mouse growth and survival by repressing the p16Ink4a and p19Arf senescence pathways. Genes Dev 2005;19:1432-1437.

12 Park I-k, Qian D, Kiel M, Becker MW, Pihalja M, Weissman IL, Morrison SJ, Clarke MF: Bmi-1 is required for maintenance of adult self-renewing haematopoietic stem cells. Nature 2003;423:302-305.

13 Zhang S, Li D, Li E, Li L, Wang J, Wang C, Lu J, Zhang X: Expression localization of Bmi1 in mice testis. Mol Cell Endocrinol 2008;287:47-56.

14 Zacharek SJ, Fillmore CM, Lau AN, Gludish DW, Chou A, Ho JW, Zamponi R, Gazit R, Bock C, Jager N, Smith ZD, Kim TM, Saunders AH, Wong J, Lee JH, Roach RR, Rossi DJ, Meissner A, Gimelbrant AA, Park PJ, Kim CF: Lung stem cell self-renewal relies on Bmi1-dependent control of expression at imprinted loci. Cell Stem Cell 2011;9:272-281.

15 Chiba T, Seki A, Aoki R, Ichikawa H, Negishi M, Miyagi S, Oguro H, Saraya A, Kamiya A, Nakauchi H, Yokosuka O, Iwama A: Bmi1 promotes hepatic stem cell expansion and tumorigenicity in both Ink4a/Arf-dependent and -independent manners in mice. Hepatology 2010;52:1111-1123. 
16 Sangiorgi E, Capecchi MR: Bmi1 is expressed in vivo in intestinal stem cells. Nat Genet 2008;40:915-920.

17 Siddique HR, Saleem M: Role of Bmi1, a stem cell factor, in cancer recurrence and chemoresistance: Preclinical and clinical evidences. Stem cells 2012;30:372-378.

18 Vandamme J, Volkel P, Rosnoblet C, Le Faou P, Angrand PO: Interaction proteomics analysis of polycomb proteins defines distinct PRC1 complexes in mammalian cells. Mol Cell Proteomics 2011;10:M110 002642.

19 Akasaka T, Takahashi N, Suzuki M, Koseki H, Bodmer R, Koga H: MBLR, a new RING finger protein resembling mammalian Polycomb gene products, is regulated by cell cycle-dependent phosphorylation. Genes Cells 2002;7:835-850.

20 Lee MG, Norman J, Shilatifard A, Shiekhattar R: Physical and functional association of a trimethyl H3K4 demethylase and Ring6a/MBLR, a polycomb-like protein. Cell 2007;128:877-887.

-21 Sun J, Lin Y, Wu J: Long non-coding RNA expression profiling of mouse testis during postnatal development. PloS one 2013;8:e75750.

22 Koeberle A, Shindou H, Harayama T, Yuki K, Shimizu T: Polyunsaturated fatty acids are incorporated into maturating male mouse germ cells by lysophosphatidic acid acyltransferase 3. FASEB J 2012;26:169-180.

23 Hofmann MC, Hess RA, Goldberg E, Millan JL: Immortalized germ cells undergo meiosis in vitro. Proc Natl Acad Sci U S A 1994;91:5533-5537.

24 Hofmann MC, Abramian D, Millán JL: A haploid and a diploid cell cycle coexist in an in vitro immortalized spermatogenic cell line. Dev Genet 1995;16:119-127.

25 Cartharius K, Frech K, Grote K, Klocke B, Haltmeier M, Klingenhoff A, Frisch M, Bayerlein M, Werner T: Matinspector and beyond: Promoter analysis based on transcription factor binding sites. Bioinformatics 2005;21:2933-2942.

26 Li B, Nair M, Mackay DR, Bilanchone V, Hu M, Fallahi M, Song H, Dai Q Cohen PE, Dai X: Ovol1 regulates meiotic pachytene progression during spermatogenesis by repressing Id2 expression. Development 2005;132:1463-1473.

-27 Hobbs RM, Seandel M, Falciatori I, Rafii S, Pandolfi PP: Plzf regulates germline progenitor self-renewal by opposing mTORC1. Cell 2010;142:468-479.

28 Martin PJ, Delmotte M-H, Formstecher P, Lefebvre P: Plzf is a negative regulator of retinoic acid receptor transcriptional activity. Nucl Recept 2003;1:6.

-29 Filipponi D, Hobbs RM, Ottolenghi S, Rossi P, Jannini EA, Pandolfi PP, Dolci S: Repression of kit expression by Plzf in germ cells. Mol Cell Biol 2007;27:6770-6781.

-30 Brawand D, Soumillon M, Necsulea A, Julien P, Csardi G, Harrigan P, Weier M, Liechti A, Aximu-Petri A, Kircher M, Albert FW, Zeller U, Khaitovich P, Grutzner F, Bergmann S, Nielsen R, Paabo S, Kaessmann H: The evolution of gene expression levels in mammalian organs. Nature 2011;478:343-348.

-31 Cho JH, Dimri M, Dimri GP: A positive feedback loop regulates the expression of polycomb group protein BMI1 via WNT signaling pathway. J Biol Chem 2013;288:3406-3418.

32 Liu S, Dontu G, Mantle ID, Patel S, Ahn NS, Jackson KW, Suri P, Wicha MS: Hedgehog signaling and Bmi-1 regulate self-renewal of normal and malignant human mammary stem cells. Cancer Res 2006;66:60636071.

33 Gargiulo G, Cesaroni M, Serresi M, de Vries N, Hulsman D, Bruggeman SW, Lancini C, van Lohuizen M: In vivo rnai screen for Bmi1 targets identifies TGF- $\beta$ /BMP-ER stress pathways as key regulators of neural-and malignant glioma-stem cell homeostasis. Cancer cell 2013;23:660-676.

34 Won H-Y, Lee J-Y, Shin D-H, Park J-H, Nam J-S, Kim H-C, Kong G: Loss of Mel-18 enhances breast cancer stem cell activity and tumorigenicity through activating notch signaling mediated by the Wnt/TCF pathway. FASEB J 2012;26:5002-5013.

35 Yu Q, Wu J: Involvement of cyclins in mammalian spermatogenesis. Mol Cell Biochem 2008;315:17-24.

-36 Zindy F, den Besten W, Chen B, Rehg JE, Latres E, Barbacid M, Pollard JW, Sherr CJ, Cohen PE, Roussel MF: Control of spermatogenesis in mice by the cyclin D-dependent kinase inhibitors p18(Ink4c) and p19(Ink4d). Mol Cell Biol 2001;21:3244-3255.

37 Bellve AR, Cavicchia J, Millette CF, O'Brien DA, Bhatnagar Y, Dym M: Spermatogenic cells of the prepuberal mouse: Isolation and morphological characterization. J Cell Biol 1977;74:68-85.

38 Dix DJ, Allen JW, Collins BW, Mori C, Nakamura N, Poorman-Allen P, Goulding EH, Eddy EM: Targeted gene disruption of Hsp70-2 results in failed meiosis, germ cell apoptosis, and male infertility. Proc Natl Acad Sci U S A 1996;93:3264-3268. 